\title{
Dynamics of the walking machines
}

\author{
Dinel POPA ${ }^{1^{*}}$, Nicolae-Doru STANESCU ${ }^{1}$, Claudia Mari POPA ${ }^{2}$ \\ ${ }^{1}$ University of Pitesti, Faculty of Mechanical and Technology, Targul din Vale str, \\ no. 1, 110040, Pitesti, Arges, Romania \\ ${ }^{2}$ Technical College Armand Călinescu, Piteşti, Arges, Romania \\ ${ }^{*}$ Corresponding author e-mail: dinel_popa@yahoo.com \\ Article history \\ Received 11.06.2017 \\ Accepted 24.09.2017 \\ DOI https://doi.org/10.26825/bup.ar.2017.008
}

\begin{abstract}
In this paper we analyze the mechanisms which can be implemented on a structure of walking vehicle, vehicle which possesses mobility and stability on different types of soils with obstacles (even other than terrestrial). We present a mechanical model for a walking self-propelled mechanism with joints, for which we perform the kinematic and dynamic analysis. For the dynamic analysis the elements of the mechanism are modeled with solids in AutoCAD, obtaining thus the geometric and mechanic properties of the self-propelled structure. We adopt a dynamic model for which, with the aid of the determined mechanical parameters, we obtain the equation of motion of the machine. After the solving of the equation of motion one obtains the variation of the angular velocity at the driving element. In the end of the paper the authors present the conclusion resulted from the performed study.
\end{abstract}

\section{Introduction}

The field of the walking mechanisms knew a special development when the people started to explore hard accessible environments. The studies show that the mechanisms based on legs, comparing to those based on wheels, are more efficient for displacements on difficult terrains.

The attention of the researchers was focused to the analysis of the locomotion by walking, specially by analyzing the walk of different species adapted to their environment, such as: the man, animals, birds, reptiles, myriapods etc. The researches in the field are numerous, different prototypes and studies being realized. In the last years one observes an increase of the researchers' interest in the 
realization of the mechanical systems forthcoming to the walking vehicles, with the simplest mechanical structures, but possessing a high degree of mobility, stability and energetic efficiency. During the history the field of the walking mechanisms was the field of research of many savants, researchers and specialists. Many researches were technical achievements in the field of mechanical toys, some of them being patented. In 1733 Maillard patented, at the French Academy of Sciences, a mechanical toys that imitated the walk of a horse, the patented mechanism being based on a mechanism with oscillating sieve. In 1878 at the Exposition in Paris, and in 1893 at Chicago, P. L. Tchebyshev presents the dummies of two mechanisms, one of them imitating the walk of the horse.

In [1] is presented a walking mechanism of Tchebyshev type. The mathematician P. L. Tchebyshev (1821 - 1894) had remarkable achievements in the synthesis of the planar mechanisms, when a point of an elements must describe a certain curve.

With more than one decade before "Star Wars" people imagined and realized walking machines. At the exposition "U. S. Army Transportation Museum" is presented a pedipulator realized by General Electric in the 1960's and named "Walking Truck" for the American army.

The machine is designed to pass dense vegetations, fallen tress, abrupt bents and to transport a payload of $500 \mathrm{~kg}$. Then, the project was abandoned because of the great costs of maintenance.

But the idea was nor abandoned. Recently, the American army tested a "fighting robot dog" named LS3. The autonomous mechanism follows the soldiers and can transport $200 \mathrm{~kg}$ of equipment on a distance up to $32 \mathrm{~km}$. It is used on difficult terrains and may relate to the soldiers.

People also imagined and realized autonomous walking machines able to displace on the soils of the planets which will be explored in the future.

Albeit they do not move on wheels or tracks, we may include such walking machines in the automotive category because they are self-propelled, move on soil and can be maneuvered.

Although in the case of the DEX definition of automotive "self-propelled vehicle used for the transport of passenger or materials " we may include the walking machines in this category.

Further on, we will present a walking mechanism which consists in four-bar mechanisms, also named Tchebyshev mechanisms.

We also discuss the dynamic analysis of the walking mechanisms which is different comparing to that of an automotive with tracks because of machine's particularities.

\section{The mechanical model of the jointed walking mechanism}

The mechanism presented in [1] is based on a four-bar mechanism the dimensions of which are obtained by synthesis, so that a point of the tiller describes a segment of a straight line. Simultaneously, in the same period of time, the body of the horse moves forward and, when the end of the tiller describes an arc of a curve, the horse arises the leg.

The Tchebyshev horse mechanism analyzed in [1] consists in four four-bar jointed mechanisms; two mechanism which model the legs on one side are sin-phased, while the other two, from the opposite side, are phase-shifted with 180 degrees relative to the first two. The main disadvantage of the mechanism is its impossibility to change the direction and avoid the obstacles.

Further on, we present the mechanical model that permits not only the linear displacement, but also the lateral displacement and the possibility to avoid the obstacles.

The kinematic schema of such a mechanism is given in Figure 1.

The mechanism is put into motion by the worm gear 1 rigidly linked to rotation with an electric motor of variable angular velocity. This puts into motion the gear 2 rigidly linked to rotation with the axis I and the conical gear 3 . The motion thus transmits to the four-bar mechanism $A_{1} B_{1} C_{1} D_{1}$. The axes I and II are common to the four-bar mechanisms 1 and 3, the tillers of which are phaseshifted with $180^{\circ}$. Further on, the motion transmits from the conical gear 3 to the conical gear 4 , rigidly linked to rotation with the bi-cardan mechanism 7 . 


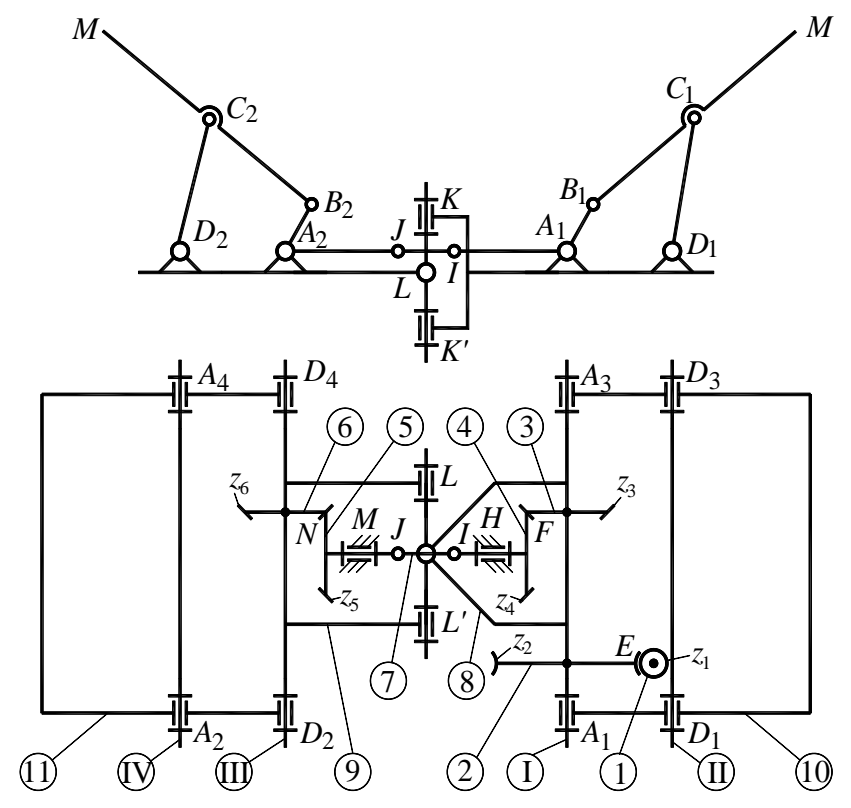

Figure 1. The kinematic schema of a jointed walking mechanism.

From the second cross of the bi-cardan mechanism the motion transmits to the conical gear consisting in the conical gears 5 and 6 . The gear 6 is rigidly linked at rotation with the axis III. In this way, the rotation motion is transmitted from the axis I to the axis III.

The axis III, at its row, puts into motion the two four-bar mechanisms 2 and 4. The four-bar mechanism 2 is sin-phased with the four-bar mechanism 1, while the four-bar mechanism 4 has the tiller phase-shifted with $180^{\circ}$ relative to the tiller of the four-bar mechanism 2.

Thus, one realized a mechanism having the same functions with that described in [1], but with the possibility of rotation around the axis LL' and around the axis KK'. This thing is possible due to the cardan formed by the forks 9 and 10 with the joints LL' and KK', respectively.

In this way, the front frame 11 may be rotated around the two axes relative to the rear frame 10 which contains the electric motor and the power supply.

Thus, the mechanism can change the direction and arise the legs of the mechanisms 2 and 4 . This thing permits it to bend in, to pass over the obstacles, to climb stairs of some certain dimensions etc.

\section{Kinematic analysis of the mechanism}

In Fig. 2 are represented the kinematic schemas for the four four-bar mechanisms.

All the four-bar mechanisms have the same dimensions of the elements:

$$
\begin{array}{lll}
A_{i} B_{i}=70 \mathrm{~mm} & B_{i} C_{i}=C_{i} M_{i}=C_{i} D_{i}=175 \mathrm{~mm} & A_{i} D_{i}=140 \mathrm{~mm} \\
i=1 \div 4 & A_{1} A_{2}=A_{3} A_{4}=460 \mathrm{~mm} & A_{1} A_{3}=A_{2} A_{4}=240 \mathrm{~mm}
\end{array}
$$

The kinematic analysis will be performed only for two four-bar mechanisms: $A_{1} B_{1} C_{1} D_{1}$ and $\mathrm{A}_{2} \mathrm{~B}_{2} \mathrm{C}_{2} \mathrm{D}_{2}$.

In [1] was presented the kinematic analysis for a four-bar mechanism by using the method of the contour's projection, and in [2] was presented the kinematic analysis for the same four-bar mechanism by using a CAD soft with AutoLisp functions.

If we choose the reference system $x A_{1} y$ (Fig. 2) and perform the kinematic analysis for the fourbar mechanism $1, A_{1} B_{1} C_{1} D_{1}$, where the element $A_{1} B_{1}$ is the driving element and has the position angle $\varphi_{1}$ with the horizontal, then the analytic calculation relations hold true for the rest of the three four-bar mechanisms too. 


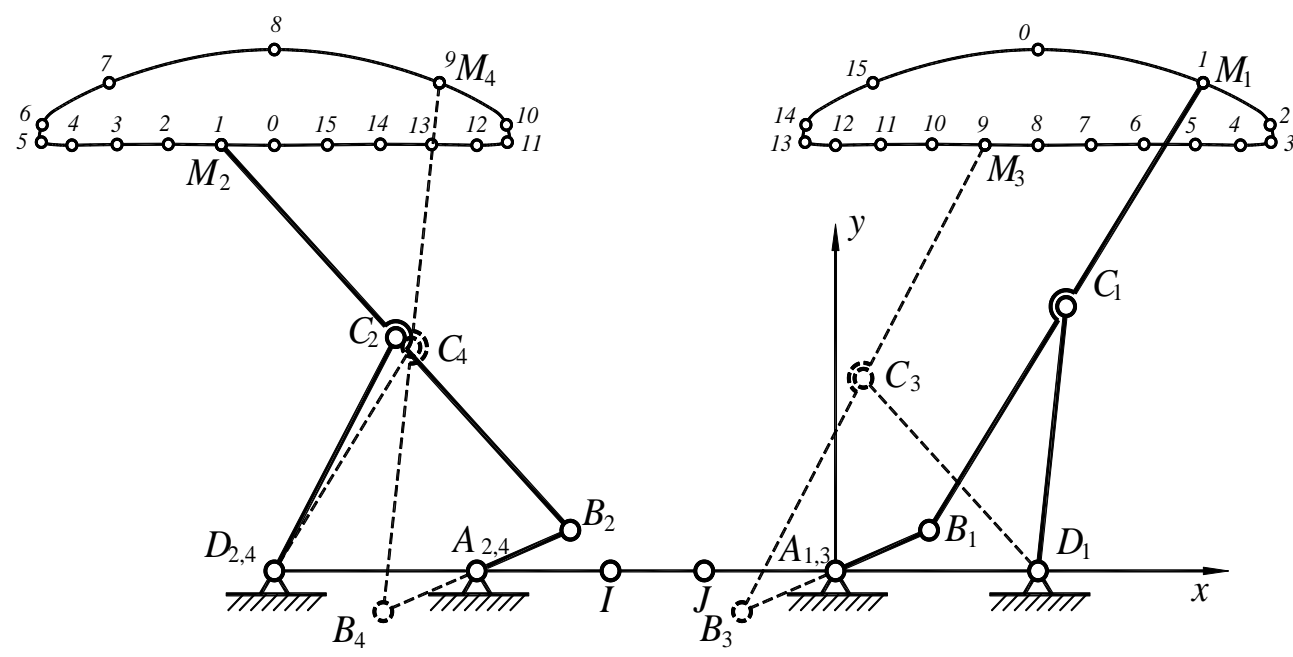

Figure 2. The kinematic schema of the four-bar mechanisms and the tiller curves

For the four-bar mechanism number $3, A_{3} B_{3} C_{3} D_{3}$, the position $\varphi_{3}$ of the driving element $A_{3} B_{3}$ is phase-shifted with $180^{\circ}$ relative to the driving element $A_{1} B_{1}$ of the four-bar mechanism 1 ( $\left.\varphi_{3}=\varphi_{1}+\pi\right)$, the rest of the analytic relations being identical.

In the case of the four-bar mechanism 2, $A_{2} B_{2} C_{2} D_{2}$, in the analytic calculation relations established for the four-bar mechanism 1 , one introduces new values only for the coordinates of the joints $A_{2}$ and $D_{2}$ relative to the system of coordinates $x A_{1} y$. For the driving element $A_{2} B_{2}$ the position angle relative to the axis $A_{1} x$ is equal to the position angle of the driving element of the four-bar mechanism $1, \varphi_{2}=\varphi_{1}$.

The kinematic analysis of the four-bar mechanism $4, A_{4} B_{4} C_{4} D_{4}$, is identical to that of the four-bar mechanism 2, the different being that the position angle $\varphi_{4}$ of the driving element $A_{4} B_{4}$ is phaseshifted with $180^{\circ}$ relative to the position of the driving element $A_{2} B_{2}$ of the four-bar mechanism 2 $\left(\varphi_{4}=\varphi_{2}+\pi\right)$.

Relative to an axis $A_{1} z$ perpendicular to the plane of figure, the four-bar mechanisms 3 and 4 are displaced with the distances $A_{1} A_{3}=A_{2} A_{4}$.

Due to the fact that the kinematic analysis of the four-bar mechanism is very known, it will be not presented in this paper.

To be out to obtain numerical results we used the analytic calculation relation from [1]. Based on these relations we wrote a calculation program in Turbo Pascal from which we obtained, in matrix form, numerical values for the coordinates of the joints of the mechanisms. The matrix assigned to the coordinate of one point has four columns, one column for each mechanism, and 360 rows (360 positions of the driving element). Thus, the abscissas of the point $M$ are $x_{M}[i, j]$, where $i=1 . . .360$ and $j=1 \ldots 4$.

In Figure 2, we over the kinematic schemas of the mechanisms we have drawn the trajectories of the point $M$ (the tiller curves). These curves were drawn in AutoCAD based on some script files generated by the realized calculation program. In Figs. 3 and 4 we represented the graphics of variation of the angular velocities of the elements $B_{j} M_{j}$ and $C_{j} D_{j}$, while in Figs. 5 and 6 we have drawn the graphics of variation of the linear velocity along the axes $x$ and $y$ of the point $M$ for the four mechanisms.

The elements were denoted as follows: $A_{j} B_{j}$ by $1, B_{j} C_{j}$ by 2 and $C_{j} D_{j}$ by 3 . The angular velocity of the element $A_{j} B_{j}$ was consider equal to $10 \mathrm{rad} / \mathrm{s}=\mathrm{ct}$. 


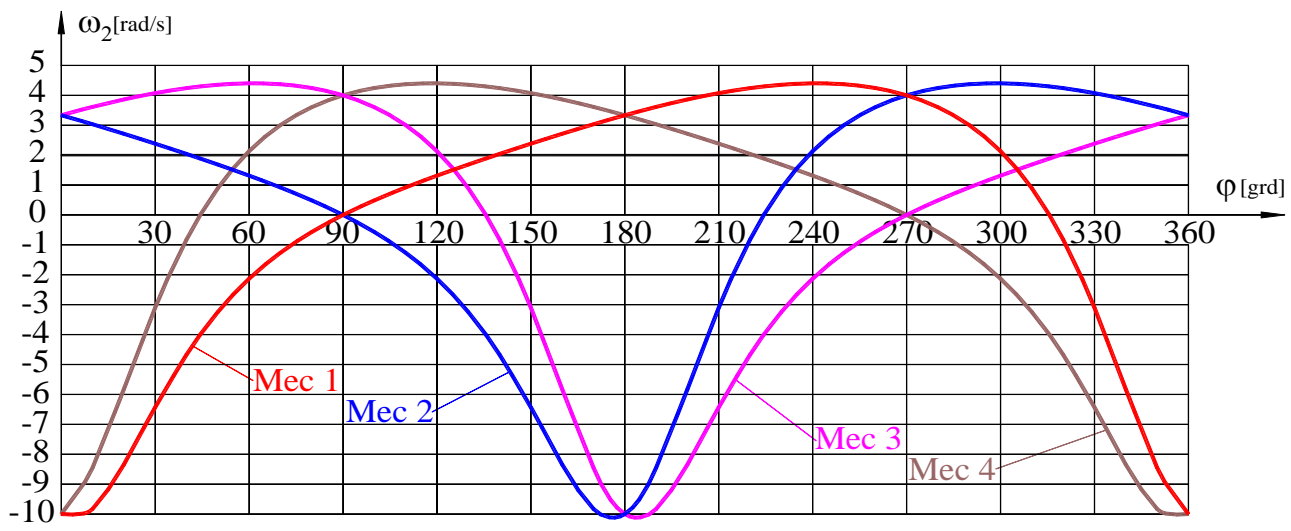

Figure 3. The graphics of variation of the angular velocities $\omega_{2}(\varphi)$ for the four mechanisms.

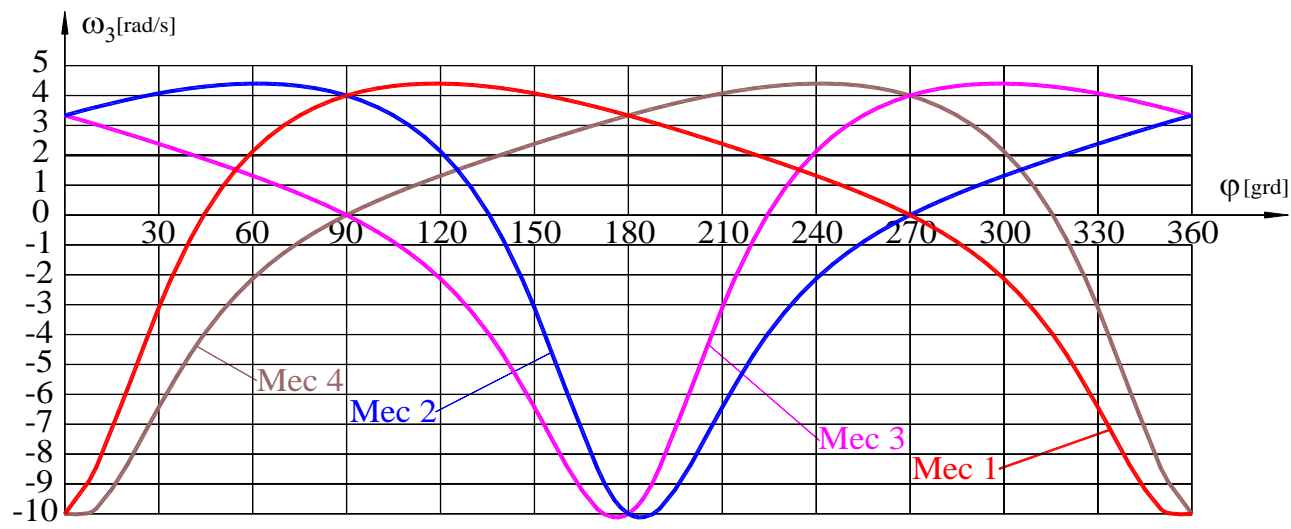

Figure 4. The graphics of variation of the angular velocities $\omega_{3}(\varphi)$ for the four mechanisms.

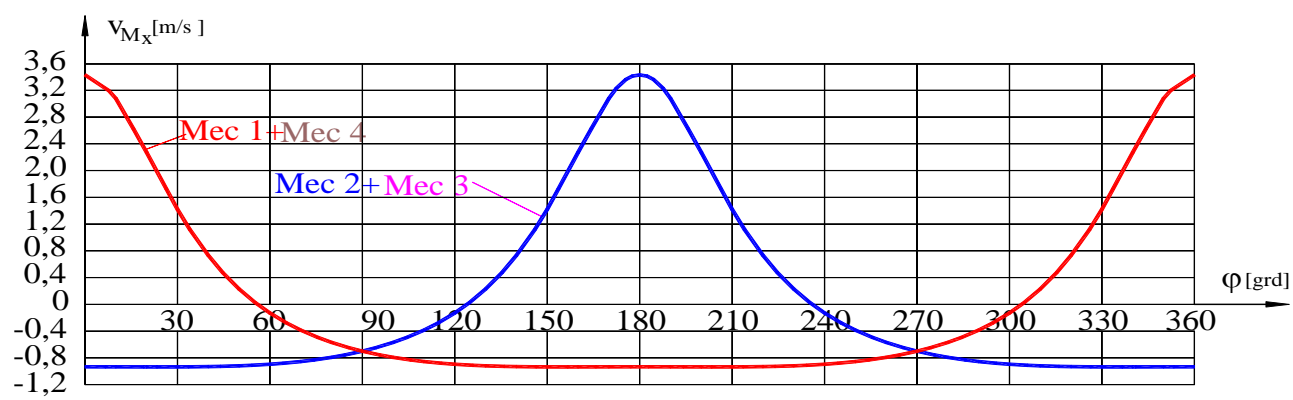

Figure 5. The graphics of variation of the velocity $M, v_{M x}(\varphi)$, for the four mechanisms

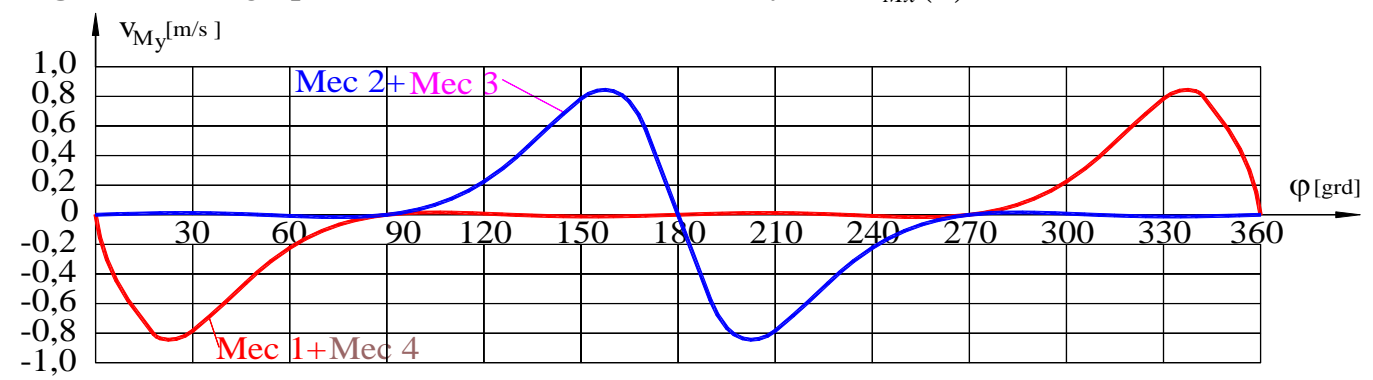

Figure 6. The graphics of variation of the velocity $M, v_{M y}(\varphi)$, for the four mechanisms.

\section{Modeling of the jointed walking mechanism}

To be out to obtain the mechanical parameters necessary to the dynamic calculation, we modeled with solids in AutoCAD the elements of the mechanism. 
After their generation one obtains the mechanical parameters with the aid of the AutoCAD command MASSPROP. AutoCAD works with units of length, and the density has the generic value 1 .

For this reason, the values returned by AutoCAD must be corrected in order to obtain the real values for the mass and moment of inertia in the International System. For instance, if one works in $\mathrm{mm}$, and the material used is the steel with the density $7800 \mathrm{~kg} / \mathrm{m}^{3}$, then the value returned by AutoCAD is multiplied by $7.8 \cdot 10^{-6}$ for the mass, and by $7.8 \cdot 10^{-12}$ for the inertial moment.

Modeling of the bars of the mechanism. The bars of the mechanism are made from rectangular bars of duralumin of $25 \times 25 \mathrm{~mm}$. Their modeling in AutoCAD is made by extrusion. One chooses the systems of coordinates as follows: in the rotation axis ( $A$ and $D)$ for the elements 1 and 3 which have rotational motion and in the center of mass $(C)$ for the element 2 . The following values are obtained:

$$
\begin{array}{ll}
m_{1}=9845.1349 \cdot 3 \cdot 10^{-6}=0.0295 \mathrm{~kg}, & J_{1}=19390538.249 \cdot 3 \cdot 10^{-12}=5.817 \cdot 10^{-5} \mathrm{kgm}^{2}, \\
m_{2}=42565.4880 \cdot 3 \cdot 10^{-6}=0.1277 \mathrm{~kg}, & J_{2}=4695663924745 \cdot 3 \cdot 10^{-12}=1.408 \cdot 10^{-3} \mathrm{kgm}^{2}, \\
m_{3}=24627.3685 \cdot 3 \cdot 10^{-6}=0.0738 \mathrm{~kg}, & J_{3}=2454690374399 \cdot 3 \cdot 10^{-12}=7.364 \cdot 10^{-4} \mathrm{kgm}^{2} .
\end{array}
$$

Modeling of the frames of the mechanism. The rear and front frames are made from duralumin, rectangular bar with the dimensions $60 \times 8 \mathrm{~mm}$, the external dimensions $60 \times 234 \times 420 \mathrm{~mm}$. One obtains the following values for the front and rear frames: $m_{F F}=493131.3279 \cdot 3 \cdot 10^{-6}=1.4794 \mathrm{~kg}, m_{R F}=4931313279 \cdot 3 \cdot 10^{-6}=1.4794 \mathrm{~kg}$.

Modeling of the axes of the mechanism. The axes of the mechanism, the number of which is four, are circular, with the diameter of $12 \mathrm{~mm}$ and the length $300 \mathrm{~mm}$. On these axes one puts the elements of the mechanism, the driving system and the gears. For one axis we get $m_{B \Phi 12}=33929.2007 \cdot 7.8 \cdot 10^{-6}=0.2646 \mathrm{~kg}$.

Modeling of the joints of the mechanism. The joints of the mechanism are rotational and prismatic ones. The revolute joints are divided into two categories. The first category is that which realizes the mobile linkage between the bars of the mechanism. The second category of revolute joints is represented by the radial axial bearings. We used: 12 bearings with collar of $12 \mathrm{~mm}, 2$ bearings in frame with foot of $12 \mathrm{~mm}, 16$ linear bearings in frame type SC8UU. Their total mass is $m_{T_{-} \text {Bearings }}=1.9 \mathrm{~kg}$.

Modeling of the gears. The gears are modeled with the AutoLisp functions described in [1]. The gears are spur conical gears, the angle of the half-cone being equal to $45^{\circ}$, the transmission ratio being $1: 1, m=2 \mathrm{~kg}, z_{1}=22$ teeth, $z_{2}=22$ teeth. One obtained the following values: $m_{\text {gear }}=21913.3485 \cdot 7.8 \cdot 10^{-6}=0.1709 \mathrm{~kg}, J_{\text {gear }}=87787964378 \cdot 3 \cdot 10^{-12}=6.847 \cdot 10^{-5} \mathrm{kgm}^{2}$. Modeling of legs. The legs are made from two bars of steel of diameter equal to $8 \mathrm{~mm}$ and length of $600 \mathrm{~mm}$. At the part of contact with the soil were attached some elements of stratified wood with the dimension $400 \times 300 \times 12 \mathrm{~mm}$. For the rigidity, the bottom part was reinforced with circular blocks of wood of diameter $33 \mathrm{~mm}$ and length $180 \mathrm{~mm}$. After the modeling, one obtains for a leg the mass $m_{L}=1.553 \mathrm{~kg}$.

The legs, having only translational motion, we added, on each edge, a system of two horizontal axes which permit these motions. A system contains two radial axial linear bearings in frame, SC8UU, mounted at $90^{\circ}$ in a common frame. In this way, one allows the longitudinal and transversal translation along two grinded axes with diameter $8 \mathrm{~mm}$ and length $500 \mathrm{~mm}$. There are 8 axes of this kind, each one having the mass $m_{\text {axis } \Phi 8}=21111.5026 \cdot 7.8 \cdot 10^{-6}=0.1646 \mathrm{~kg}$.

These axes are linked to the two frames by another two frames made of rectangular bar with the masses $m_{F 1}=324120 \cdot 3 \cdot 10^{-6}=0.97236 \mathrm{~kg}, m_{F 2}=324120 \cdot 3 \cdot 10^{-6}=0.97236 \mathrm{~kg}$.

Modeling of the linkage. The linkage must permit the bending in of the mechanism and the lift of the frame in order to pass the obstacles and, eventually, to climb the stairs. We designed the linkage 
which is rigidly assembled between the two frames. The linkage is made from platband with the thick of $5 \mathrm{~mm}$. The assembling of the previous elements is made by welding. The mass of the system is $m_{S C}=8003648853 \cdot 3 \cdot 10^{-6}=2.401 \mathrm{~kg}$.

Mounted between the two frames it permits the bending in of the mechanism. If the linkage rotates with $90^{\circ}$, then it will permit the lift of the front frame relative to the driving rear frame. The actuation of the linkage is assured by two pinion-gear mechanisms acted by an electric motor of $12 \mathrm{~V}$ and a reducer. Two such systems disposed at $90^{\circ}$ permit the two motions.

The mass of the cardan that transmits the motion from the rear frame to the front one is $m_{\text {Cardan }}=0.465 \mathrm{~kg}$, and the moment of inertia has the value $J_{\text {Cardan }}=8.557 \cdot 10^{-5} \mathrm{kgm}^{2}$.

Modeling of the moto-reducer. The walking mechanism is acted by an electric motor with wormgear reducer. Its mass is $m_{\text {Mot }+ \text { red }}=3.4 \mathrm{~kg}$.

Finally, after the assembling, it will result the photographic representation in Figure 7.

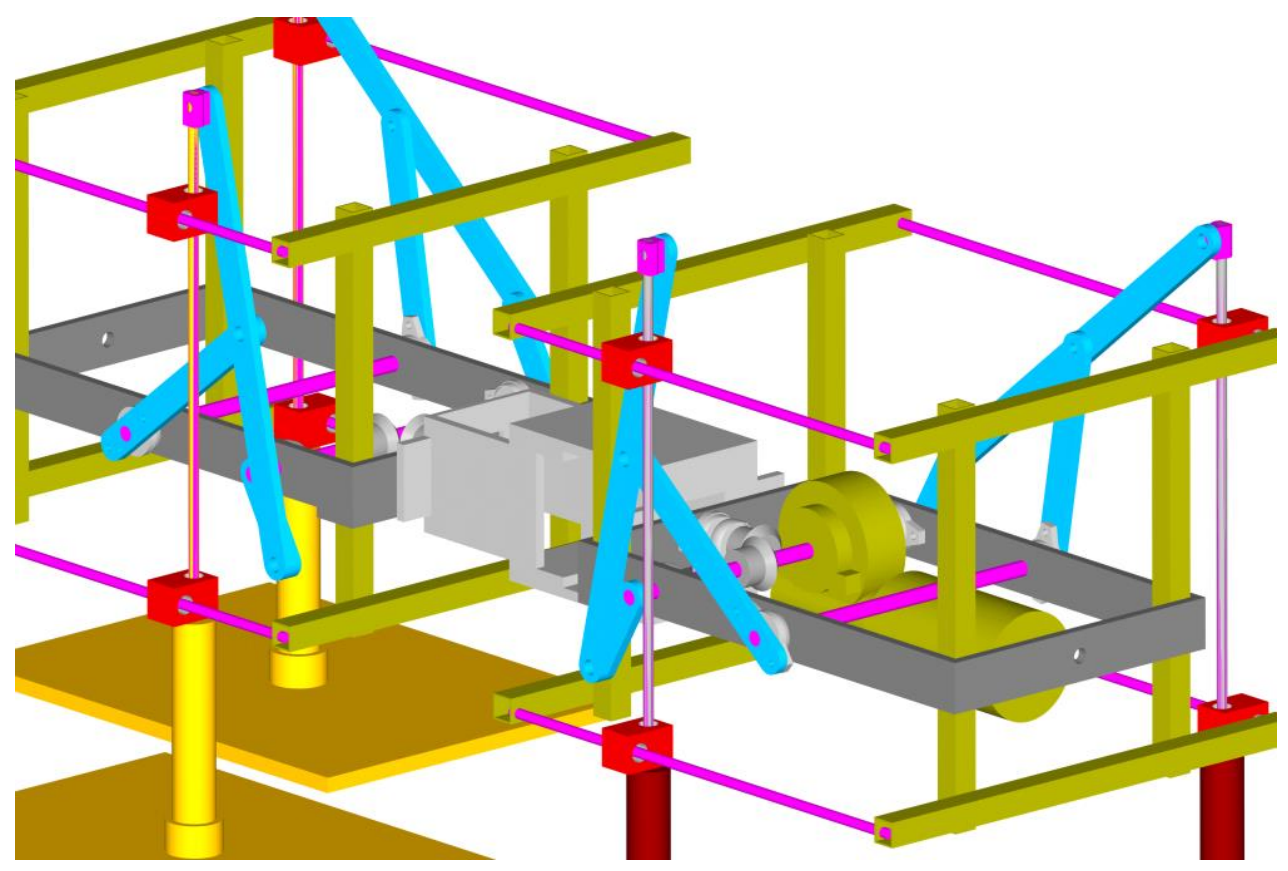

Figure 7. Photographic representation of the jointed walking mechanism.

\section{Dynamic analysis of the walking machines}

The goal of the dynamic analysis is to determine the variation of the angular velocity at the driving element under the influence of the forces and torques acting the elements.

To be out to do this, a method consists in choosing a simple dynamic model which replaces, from the energetic point of view, the machine.

We purpose the reduction at the driving element and we choose as dynamic model [1] a bar in rotational motion and having as dynamic parameters $J_{r e d_{1}}$ and $M_{r e d_{1}}$. These parameters are function of the position $\varphi$ of the driving element. Since the kinematic analysis was performed with an angular step $\Delta \varphi=1^{\circ}$, the dynamic parameters are obtained as vectors with 360 values: $J_{\text {red }_{1}}[i]$, $M_{r e d_{1}}[i]$ with $i=1 . . .360$.

The moment of inertia reduced at the driving element $J_{r e d_{1}}$ has the expression

$$
J_{\text {red }}[i]=\frac{1}{\omega_{1}^{2}} \sum_{j=1}^{4}\left(J_{1} \omega_{1}^{2}+J_{2} \omega_{2 j}^{2}+m_{2} v_{C j}^{2}+J_{3} \omega_{3 j}^{2}+\frac{m_{\text {frame }}}{2} v_{M j}^{2}\right)+J_{\text {cardan }}+4 J_{\text {gear }}+2 J_{B \Phi 12},
$$


while the reduced motor torque $M_{\text {red }_{1}}$ has the expression

$$
M_{r e d_{1}}[i]=\frac{1}{\omega_{1}} \sum_{j=1}^{4}\left(-G_{1} v_{C_{1 y} j}-G_{2} v_{C_{y} j}-G_{3} v_{C_{3 y} j}-G_{P} v_{M_{y} j}\right)
$$

We used the notations:

- $\omega_{i j}$ - the angular velocity of the element $i$ of the mechanism counted as $j$,

$-v_{M j}$ - the velocity of the point $M$ of the mechanism $j$,

- $v_{C_{i y} j}$ - the velocity of the center of mass of the element $i$ of the mechanism $j$,

$-v_{M_{y} j}$ - the velocity along the axis $y$ of the point $M$ of the mechanism $j$,

$$
\begin{gathered}
m_{\text {frame }}=4\left(m_{1}+m_{2}+m_{3}\right)+m_{F F}+m_{R F}+m_{F 1}+m_{F 2}+4 \cdot m_{B \Phi 12}+8 \cdot m_{\text {axis } \Phi 8}+4 \cdot m_{\text {gear }} \\
+m_{S C}+m_{T_{-} \text {Bearings }}+m_{\text {Cardan }}+m_{\text {battery }} .
\end{gathered}
$$

For the battery we considered the mass $m_{\text {battery }}=2.5 \mathrm{~kg}$.

The integration of the moving equation is performed for the regime phase when $M_{\text {red }_{1}}$ is function only of position $\left(M_{\text {red }_{1}}=M_{r e d_{1}}(\varphi)\right)$, and the work produced by it on a dynamic cycle $\varphi_{d}=2 \pi$ is equal to zero,

$$
\int_{0}^{\varphi_{d}} M_{r e d_{1}}(\varphi) \mathrm{d} \varphi=0
$$

One also knows the value of the average angular velocity, $\omega_{m}=\omega_{1}$, as an integral of the angular velocities $\omega(\varphi)$,

$$
\omega_{m}=\frac{1}{\varphi_{d}} \int_{0}^{\varphi_{d}} \omega(\varphi) \mathrm{d} \varphi .
$$

Denoting the work by $L(\varphi)=\int_{0}^{\varphi} M_{\text {red }}(\varphi) \mathrm{d} \varphi$ and $J_{0}=J_{\text {red }_{1}}(0)$, and applying the theorem of the kinetic energy, we obtain the expression of the variation of the angular velocity

$$
\omega=\sqrt{\frac{2 \cdot L(\varphi)+J_{0} \omega_{0}^{2}}{J_{\text {red }_{1}}(\varphi)}} .
$$

In relation (6) one does not know the value of the angular velocity $\omega_{0}$, but it can be determine from the equation

$$
\omega_{m}=\frac{1}{\varphi_{d}} \int_{0}^{\varphi_{d}} \sqrt{\frac{2 L(\varphi)+J_{0} \omega_{0}^{2}}{J_{r e d}(\varphi)}} \mathrm{d} \varphi
$$

The solving of the equation (7) is not an easy task, especially when $M_{\text {red }_{1}}$ and $J_{r e d_{1}}$ are given as tables with values (vectors with 360 positions). In [1] the authors purpose an iterative method to determine the value of the angular velocity $\omega_{0}$. Applying this method, we obtain, with an imposed precision of $10^{-3} \mathrm{rad} / \mathrm{s}$, the value of $\omega_{0}$; introducing this value in the relation (6), we get the variation of the angular velocity at the driving element in the regime phase. 
The calculation program, written for the kinematic analysis, is completed now with the values of the masses and the values of the moments of inertia, values determined for all the elements, using the calculation relations (1)-(3) and the procedure of solving the equation of motion, presented in [1].

The procedure of solving the moving equation determines the active torque necessary for the actuation of the mechanism. In the case of the previous numerical values one obtains $M_{a c t}=14.492 \mathrm{Nm}$.

The calculation program also realizes a script file with the aid of which, in AutoCAD, one may draw the graphic of the variation of the angular velocity in Figure 8.

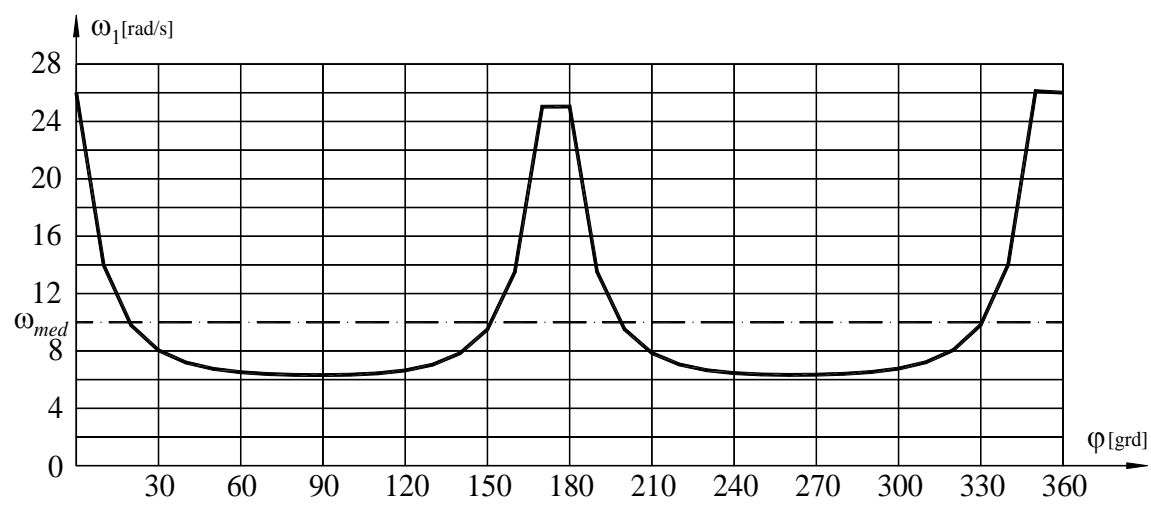

Figure 8. The variation of the angular velocity at the driving element.

\section{Conclusions}

The self-propelled walking mechanism presented here bases on four Tchebyshev- type four-bar mechanisms; two of these mechanisms are phase-shifted and two are sin-phased.

In order to bend in and avoid the obstacles, the walking machine has two frames, the rear frame with the propulsion system (moto-reducer, battery, electric cables and command system) and two four-bar mechanisms, and the front frame with another two four-bar mechanisms.

The motion is transmitted from the rear frame to the front one by a gear transmission consisting in 4 conical gears and a bi-cardan transmission. By using an angular joint of cardan-type, the walking machine can bend in, pass the obstacles and climb stairs of a certain dimension.

For the study of the dynamics of the mechanism, we modeled its elements with solids in AutoCAD, we obtain their mechanical properties, based on which, with the aid of a calculation program written in Turbo Pascal, we solved the moving equation of the machine.

The purposed dynamic model is that of a bar in rotational motion, this model corresponding, from the energetic point of view, to the walking machine.

The propelled machine can be also loaded, for its own displacement it was necessary an average active torque of $14.492 \mathrm{Nm}$. If one loads the machine, which has its own mass of approximate $28.5 \mathrm{~kg}$, with a load equal to its own weight, the active torque given by the calculation program is equal $35.233 \mathrm{Nm}$.

The walking machine presented in the paper was experimentally realized, using the specified dimensions. The experimental results are in good agreement with those determined in analytic way.

\section{References}

[1] Pandrea N, Popa D and Stănescu ND, 2017 Clasical and modern approaches in the theory of mechanisms, John Wiley \& Sons, Chichester, UK

[2] Popa D, Stănescu ND and Popa CM, 2013 Determination of the Reactions in Linkages with AutoLISP Functions, Annals of the Oradea University, Fascicle of Management and Technological Engineering 1 73-8 
[3] Popa D, Stănescu ND and Popa CM, 2013 Animation of the Vector Relations by AutoLISP Functions, Annals of the Oradea University, Fascicle of Management and Technological Engineering 1 79-84

[4] Kumar V, Schmiedeler J, Sreenivasan SV and Su HJ, 2013 Advances in Mechanisms, Robotics and Design Education and Research (Mechanisms and Machine Science), Springer

[5] Norton RL, 2003 Design of Machinery: An Introduction to the Synthesis and Analysis of Mechanisms and Machines, McGraw-Hill

[6] Artobolevski I, 1978 Les mécanismes dans la technique moderne. Tome 1-5, Ed. MIR, Moskow

[7] Sclater N, 2011 Mechanisms and mechanical devices sourcebook, Fifth Edition, McGraw-Hill

[8] Charles W and Sadler P, 1993 Kinematics and dynamics of machinery, Second Edition, Harper Collins College Publishers

[9] Ambekar AG, 2007 Mechanism and Machine Theory, Prentice-Hall

[10] You Z and Chen Y, 2011 Motion Structures: Deployable Structural Assemblies of Mechanisms, 1st Edition CRC Press

[11] Klebanov BM and Groper M, 2015 Power Mechanisms of Rotational and Cyclic Motions, CRC Press

[12] Russell K, Shen Q and Sodhi RS 2015 Kinematics and Dynamics of Mechanical Systems: Implementation in MATLAB and SimMechanics, CRC Press

[13] Lenarcic J, Bajd T and Stanisic MM, 2013 Robot Mechanisms, Springer

[14] Wu Y, Li S, Liu S and Qian Z, 2013 Vibration of Hydraulic Machinery, Springer

[15] Mostafa MA, 2012 Mechanics of Machinery, 1st Edition, CRC Press

[16] Stanisic MM, 2014 Mechanisms and Machines: Kinematics, Dynamics, and Synthesis, 1st Edition, CL Engineering

[17] Wittenburg J, 2016 Kinematics: Theory and Applications, 1st Edition, Springer

[18] Russell K, Shen Q and Sodhi RS, 2013 Mechanism Design: Visual and Programmable Approaches, CRC Press

[19] Zhao Z, Feng Z, Chu F and Ma N, 2013 Advanced Theory of Constraint and Motion Analysis for Robot Mechanisms, 1st Edition, Academic Press

[20] Sacks E and Joskowicz L, 2010 The Configuration Space Method for Kinematic Design of Mechanisms, Massachusetts Institute of Technology Press

[21] Rider MJ, 2015 Design and Analysis of Mechanisms: A Planar Approach, 1st Edition, Wiley

[22] Myszka DH, 2011 Machines and Mechanisms: Applied Kinematic Analysis, 4th Edition, Prentice Hall

[23] Uicker J, Pennock G and Shigley J, 2010 Theory of Machines and Mechanisms, 4th Edition, Oxford University Press 